\title{
WHY BENCHMARKING? UNDERSTANDING THE PROCESS
}

\section{Wasila Abdulaziz Elasheg ${ }^{1}$}

1 Assistant professor Tripoli University faculty of Engineering Petroleum department

Email: welasheg@gmail.com

\section{Published at 01/08/2021}

Accepted at 24/07/2021

\begin{abstract}
The essence of benchmarking is the process of identifying the highest standards of excellence for products, services, or processes, and then making the improvements necessary to reach those standards - commonly called "best practices". This paper is to summaries the experience of spreading the knowledge of applying Benchmarking models and detailing the steps to maximize an organization's potential for success to motivate a desire of managers to follow these steps in order to achieve best practices.
\end{abstract}




\section{1- Introduction}

Nowadays the world changes at an astonishing pace as a result of flowing technological changes and the demand of innovation, quality and speed all at once. The essence of benchmarking is the process of identifying the highest standards of excellence for products, services, or processes, and then making the improvements necessary to reach those standards - commonly called "best practices" Khurrum S. Bhutta and Faizul Huq, 1999. Benchmarking has also become absorbed as a valuable component into many other quality management systems and as Asrofah et al. (2010) stated that there are many companies, which emphasize on the importance of benchmarking, however, not many companies understand well enough about benchmarking. The lack of excitant of simplified benchmarking process is one of the main causes that make it difficult for companies to employ the tools. My goal in this paper is to summaries the experience of spreading the knowledge of applying Benchmarking models by detailing the steps to maximize an organization's potential for success to motivate a desire of managers to follow these steps in order to achieve best practices.

According to Webster's Collegiate Dictionary benchmark definition is the mark on a permanent object indicating elevation and serving as a reference in topographical surveys and tidal observations. It is a point of reference from which measurements may be made. Benchmarking is something that serves as a standard by which others may be measured. In management Benchmarking is a quality tool that allows an organization to improve organizational performance by assessing itself as well as its competitors. It enables an organization to measure its products, services and processes against those of leading companies and organization throughout the world. Through this knowledge, organization can develop and implement plans to achieve increased customer satisfaction and ultimately become the best at what they do (Ertek, Sevinç, Ulus, Köse, Şahin, 2013) It is often stated that those who benchmark do not have to reinvent the wheel (Razmi et al. 2000).

Many view Benchmarking as a method for comparing key figures, often financial key figures, for ranking the organization in relation to competitors or the industry average. This might have been the main application of benchmarking earlier, but today it is a far more powerful tool much more widely applicable. The core of the current interpretation of Benchmarking is:

- Measurement, of own and the Benchmarking partners' performance level, both for comparison and for registering improvements.

- Comparison, of performance level, processes, practices, etc.

- Learning, from the Benchmarking partners to introduce, improvement in your own organization.

- Improvement, which is the ultimate objective of any Benchmarking study. 
A 1995 research study conducted by the American Productivity \& Quality Center's (APQC) shows that benchmarking made tremendous leverage. More than 30 organizations reported an average $\$ 76$ million first year payback from their most successful benchmarking project, with $40 \%$ ranging between 1 million and 9.9 million US\$. Among the most experienced companies in Benchmarking, the average payback raise to $\$ 189$ million. Areas of greatest improvement, as revealed by the same study and as a result of benchmarking projects were: reduced costs, increased productivity and reduced cycled time of operations (Kelessidis, 2000).

Benchmarking exercises using computer simulations are routinely undertaken in the finance sector and in scientific fields, such as mechanical engineering and meteorology (Gustafson 2004). This may become more common practice in other fields as the available technology becomes more flexible and accessible. It appears from the literature that, increasingly, organizations are working with a suite of benchmarks and processes rather than focusing on a single process.

Financial sectors report using multivariate approaches and benchmarking suites or panels (Aupperle \& Dunphy 2003; Dawkins, Feeny \& Harris 2007; Lin \& Mei 2006 in Michael Cole 2011).

Public sector approaches utilize benchmarks in multiple domains of their operations, with the example of the Oregon State Government using 160 benchmarks covering economic, environmental, transportation and other areas of concern (Ford \& Hibbard 2000 in Michael Cole 2011). On the other hand, Niven 2002 (in Gustafson 2004) cautions that most organizations still focus on a single area of operations when Benchmarking, but he points out that the best-in-class organisation probably has a unique mix of a number of different activities that collectively produce an outstanding performance.

\section{2- What is Benchmarking?}

Benchmarking is the process of improving performance by continuously identifying, understanding, and adapting outstanding practices and processes found inside and outside an organization (company, public organization, University, Clusters, College, etc.). It was pioneered by Xerox Corporation in the 1979s, as part of their response to international competition in the photocopier market, and originated from reverse engineering of competitors' products. Its scope was then enlarged to include business services and processes. Xerox now benchmarks nearly 240 performance elements although, when they started benchmarking several years ago, considerably fewer elements were benchmarked (Shen et al., 2000). The systematic regulation of Benchmarking is focused on identifying, studying, analyzing, and adapting best practices and implementing the results. To consistently get the most value from the Benchmarking process, senior management may discover the need for a significant culture change. That change, however, unleashes the Benchmarking full potential to 
generate large paybacks and strategic advantage. Aupperle, KE \& Dunphy, S 2003 identifies Benchmarking as a continuous process of evaluation of products, services and practices with respect to those of the strongest competitors or of the enterprises recognized as leaders. Either the enterprise adopts these practices, or it adapts them with the aim of improving its performance. In a direct way, the Benchmarking is a process of evaluation and improvement of performance. Based on this prospective Benchmarking is a form of comparative analysis. It is necessary to establish some common ground as the basis for comparison. Usually identifies one or more functional areas for analysis and selects one or more metrics as a quantitative basis for comparison. These are then compared with agreed benchmarks derived from recognized sources of best practice.

\section{3- Why do you Benchmark?}

There are different reasons why an organization, or a company would want to Benchmark. When you Benchmark you find out who is the best, you gather actionable data for change and process improvement, and you realize that there is a world outside of your own with great ideas that you can use. According to the European Secretariat for Cluster Analysis (ESCA, www.clusteranalysis.org) Benchmarking of cluster management organization offers an efficient way to identify the potential of a cluster and to develop strategic recommendations for its further development within a short time frame.

Benchmarking can enhance an organization's performance by:

- Satisfy customer's need and expectations (you should know what is important to your customer and what will meet their expectations).

- Discuss and understand the methods and practice needed to reach new goals (you should know what is needed to reach performance excellence and how to reach it).

- Achieve superior performance (you should be performing this study to improve the performance of the organization).

- Adapt best practices (through research you should be finding best practices that can improve your process).

- Develop and stimulate strategic goals/planning (if this study does not support the strategic plan then the study should not be performed).

- Stay informed on the state - of - the- art business practice (through research you will learn best practices that are on the cutting edge).

- Encourage creative thinking - get out of the box (while performing this study you will discover different creative ideas used by other organizations).

- Review/study competitive comparisons (research and interviews will provide information for you to compare your organization to). 
- Accelerate process improvement (performing a study will provide you with information/results from others so that you can implement change quicker).

- Discover emerging technologies (research will provide you with new ways of doing things).

\section{4- What do you expect?}

Benchmarking offers the following benefits to companies and organizations:

- Highlights areas of practice and performance requiring attention and improvement.

- Identifies strengths and weaknesses to other respondents.

- Establishes Company's true position versus the rest, making thus easier for the company to raise the organizational energy for change and develop plans for action.

- Helps measure current company performance.

- Prevents reinventing the wheel (Why invest the time and costs when someone else may have done it already - and often better, cheaper, and faster?).

- Accelerates change and restructuring by using tested and proven practices, convincing skeptics who can see that it works and overcoming inertia and satisfaction and creating a sense of urgency when gaps are revealed.

- Leads to "outside the box" ideas by looking for ways to improve outside of the industry.

- Forces organizations to examine present processes, which often lead to improvement in and of itself.

- Makes implementation more likely because of involvement of process owners.

- Enables the identification of other companies and/or organizations with processes resulting in superior performance, with a view to their adoption.

\section{5-Types of Benchmarking}

There are, in general, four types of Benchmarking:

\subsection{Internal}

A process where organizations learn from "sisters" companies, divisions, or operating units. This process could be applied in organizations having multiple units (for e.g. multinationals, companies with sale offices around the country, with multiple factory locations within the same country).

Advantages \& disadvantage:

- Low cost, faster and easy to transfer lessons learned.

- Provides highest degree of process detail and simplified access to process information.

- Gain a deeper understanding of your own process. - Good practice/training with 
benchmarking process. - Low performance improvement 10\% increase (Taxes instrument, 1991).

- Limits options of growth and foster weakness.

\subsection{Competitive}

Benchmarking is performed versus competitors and data analysis is done as to what causes the superior performance of the competitor. It can be, in some respects, easier than other types of benchmarking and in some respects more difficult. It is easier in the sense that many exogenous variables affecting company performance may be the same between the source and the recipient organization, since we are talking about companies of the same sector. On the other hand it is more difficult because, due to the competitive nature, data recuperation will not be straightforward. Difficulties of this type may be overcome if the two organizations have for e.g. different geographical markets.

Advantages \& disadvantages

- Provides a strategic insight into marketplace.

- Prioritizes areas of improvement according to achievement.

- Possible partnership.

- Relatively low performance improvement about 20\% (Taxes instrument, 1991).

- Threatening and may provide misleading.

- Legal issues regarding business relation between competitors, as well as limited by "trade secrets".

\subsection{Functional (process)}

Here we look at processes, which may be similar, but in different organizations, producing different products, for e.g. airline industry \& hospital industry looking at the process of catering their 'clients'. Advantages \& disadvantages

- Better improvement 35\% (Taxes instrument, 1991).

- Takes advantage of function and professional networks to develop detailed process understanding which provide industry trend understanding.

- Quantitative comparisons and common language.

- Easy to transfer lessons learned.

- Diverse corporate culture.

- Take more time than internal and competitive Benchmarking.

\subsection{Generic}

We would look here at the technological aspects, the implementation and deployment 
of technology. How else other organizations do it? Hence the source organizations may be of same industry or from another industry.

Advantages \& disadvantages

- Provides the greatest opportunity for process breakthroughs because organization doesn't compete.

- Information is usually available.

- Innovative and there is high optional for discovery.

- High cost and Take a long time to plan known world - class companies are busy with.

- Quantum changes can bring high risk and fear.

\section{6- Benchmarking technique}

There are many approaches that can be followed in the implementation of benchmarking Deros, et al 2011. Four or five phase approach, the steps approach (Spendolini's 11 steps, Camp's Texas Instrument, Xerox's 10 steps, Coopers \& Lybrand's 9 steps) etc. The four phase approach will be explained in the following paragraph. The four phase approaches:

\subsection{Planning Phase:}

The planning phase is that will set the stage for the whole benchmarking study. It is where the process / function is selected and define by using information gathered from your customers in some type of format (i.e. focus session, survey, interviews) which will express to you their needs and expectations.

6.1.1 Steps to follow during the planning phase:

1 - Select the process and identify the objective of study.

To acknowledge that a benchmarking study is needed and that the study will focus on specific objective: - Select a process that has big impact on performance.

- Select a process that causes customer dissatisfaction.

- Select a process that is not performing up to customer standards.

- Define the specific scope of the study.

2- Identify and gain participation of the sponsor and stakeholders.

It is important to get the support and commitment from the executive leaders.

- Obtain approval from the executive leaders / staff.

- Discuss the benchmarking study with the sponsor and stakeholders and obtain commitment for resources.

- If you don't obtain commitment, rethink the idea of performing the study. Lack of 
commitment can stop the study at any time and implementation of any changes will be difficult.

3- Submit a benchmarking request to your benchmarking coordinator. It is important for you to find out what type of studies researches have been performed in your organization. In some instances a benchmarking study has already been completed on what you might tasked to do. If your organization has a benchmarking coordinator, she / he should know. Sometimes a coordinator will also be able to link you with another organization that might have performed the same or similar study.

- Contact the benchmarking coordinator to ensure this type of study has not been performed before.

- Obtain the request form (if required to register your study) and return it to the coordinator with the appropriate information and approving authority signature.

4- Select a cross-functional team that represents all aspects of the process being benchmarking. There are many variations possible in the team composition. The team composition should be carefully determined based on the scope of the effort, the available resources, and the overall objective

Team size is dependent on the scope of the project (four to eight team members usually constitutes a good working group).

- A team should be cross functional, representing all functions involved in the process.

- A team member should have some of the following attributes:

a) Process knowledge or expertise.

b) Responsibility for implementation.

c) A Stakeholder.

d) Objective.

e) Analytical

f) Flexible

g) Risk taker

h) Optimistic

i) Dependable

j) Communicator

5- Provide benchmarking awareness training to the team and to the sponsor/stakeholders to ensure that all have the same understanding of what benchmarking is and what is involved in a study.

6- Identify products / services, customers, and their expectations. Any study should be 
related to the customer, their needs and expectations, and should link back to organization strategic plan. - Identify the products and services (outputs) generated through the process being benchmarked. - Identify the customers who use the products and services. - Identify the customer's needs and expectations through focus sessions, surveys, interviews, etc.

7- Identify and select the Critical Success Factors (CSF) (the one that is important to your customer and the one that is going to make a difference if improved).

- Analyze and document the process flow with the team.

- Identify the process that has the greatest impact on performance.

- Identify activities that have the greatest impact on the customer. Convert the CSF's into specific metrics that will allow best - in - class organizations to better understand your information requirements.

8- Analyze and document the process. It is important to know your own process. The way to gain this type of information is to flowchart the process with process experts then compares it with the "Best - in - Class" organization.

- Flowchart the process being benchmarked.

- Gather process information from flow charts, face to face interviews, archival records, etc.

- Retain a comprehensive knowledge of your own process - understand the process from beginning (source of inputs) to end (who receives the output) before you talk with other organization.

- Understand how change will affect others within your organization. - Use any source of information within your own organization that could lead to obvious improvements and that can be accomplished as short term goals.

- Document your performance standards. During the flowchart process it is possible to see what steps are really necessary. Those that not necessary can be eliminated.

9- Review and establish general performance measures. It is important to have basic performance measures while performing the study so that you can easily compare your measures with others. If you don't have measures already in place, put a system in place now to start collecting data. By the time you reach phase three a significant amount of data will be collected.

- Establish basic performance measures (quality, cost, cycle time).

- Establish specific measures to allow you and your partner to speak the same language.

- Establish a baseline to serve as the foundation for comparison of improvements. 


\subsection{Collection Phase:}

$\mathrm{Al}$ the research will be performed in this phase (primary and secondary research). The secondary research is a literature review and will be $80-85 \%$ of this phase. The nine steps to follow during the collecting phase:

1- Develop criteria for secondary research. Before you start phase 2 you need as a team to discuss the scope of the study and what type of information you will be looking for in your researching process. Develop a data collection form with a scoring mechanism to indicate how each data is important to the study.

a. Notify the team with the type of data that will be needed for the study.

b. Develop standard questions and a data collection form for the team to use during literature search.

c. Develop criteria to rate prospective organizations (number of employees, workload, time constraints, company mission, size and industry).

d. Create a scoring mechanism.

2- Conduct an extensive literature review (secondary research). The literature review is where and when you find the information important to establish who is the best or who is the best you can find which is connected to the process you are researching.

a. Use secondary resources (databases, industry / trade publications, governments' agencies, annual reports, seminars / conferences, internet, networking, professional organizations, and books).

b. Document finding and share with team members.

3- Identify "Best - in - Class" and potential partners.

a. Identify best practice organizations from information gathered through the literature review.

b. Select the organizations that best fits the requirements.

c. Create a potential partner listing.

d. Create a folder on each potential partner to file appropriate information collected.

4- Review legal and ethical issues and benchmarking protocol. Review the Code of Conduct.

a. You are representing your organization each time you encounter a best - in - class organization, respect yourself well.

b. Present yourself in a professional manner.

c. Be well prepared.

d. Present yourself with confidence. 
e. Ask only information that you would be willing to share with them.

f. Establish the rules up front with your partner and treat any acquired information as confidential.

5- Plan data collection strategy for primary research. What type of method you will use to gather information? Create a mechanism.

a. Brainstorm as a team initial screening questions.

b. Test the effectiveness of your questions before using them.

c. Design a rating scheme for data collected to determine actual partners.

d. Make the appropriate preparations to contact the organizations for the purpose of validating/verifying the information you found.

6- Conduct primary research of potential partners and narrow list to actual partners. Now you have created your list of optional partners and you are well prepared by knowing the Code of Conduct it is time to start the primary research. It is time consuming process that can give you firsthand information about how actually they perform the process that you are benchmarking.

a. Prepare for initial contact (telephone script, questionnaire).

b. Contact an organization and inform them of your intentions.

c. Determine the level of interest.

d. Inform them why you believe they represent best - in - class organization.

e. Verify information found in secondary research.

f. Request their participation in the benchmarking study.

g. Set ground rules regarding eithics and protocol so that both parties can clearly understand what is expected before any information is exchanged.

h. Ask or mail preliminary questions.

i. Mail a letter of introduction.

j. Offer a copy from your final report to them in appreciation for their cooperation and assistance.

The data collected in the primary research will give you information about if the organization meets the criteria to become actual partner so you need to leave the door open for farther connection.

7- Contact actual partners and ask more in depth questions. It is important to analyze the information collected to narrow the list to start looking for more in-depth questions, a. Review responses to initial questions. 
b. Rate organization, narrow the list of optional partners to actual partners.

c. Select best organization for further dialog.

d. Decide on data gathering mechanism to be used in further dialog with organization.

e. Ask more in depth question.

f. Document the finding.

g. Determine if site visit is required.

h. Prepare and conduct site visit.

6.3 Analysis Phase

1. Compare your current performance data to your partners' data

a. Sort and compile data

b. Make your performance data comparable (normalize)

c. Identify gaps

2. Identify operational best practices and enablers

a. What are participants doing that you are not doing? Perform gap analysis.

b. How do they do it? (Enablers).

3. Formulate strategy to close the gaps

a. Project the gap to three to five years from now.

b. Define the goals that must be achieved in order to close the gap.

c. Assess adaptability of practices and enablers.

d. Identify opportunities for improvement.

e. Develop implementation plan

6.5 Adapting Improvements

1. Implement the plan

2. Monitor and report progress

3. Document the study

a. Communicate the results (internally and to benchmarking partners)

b. Assist in the internal transfer of best practices

4. Plan for continuous improvement

a. Identify new benchmarking opportunities

b. Set new goals 


\section{References}

$\begin{array}{llllll}\text { American } & \text { Productivity } \quad \& \quad \text { Quality } & \text { Center's } & \text { (APQC) } 1995\end{array}$ https://www.apqc.org/benchmarking-portal

Asrofah, T., S. Zailani and Y. Fernando, 2010. "Best practices for the effectiveness of benchmarking in the Indonesian manufacturing companies", Benchmarking. Int. J., 17: 115-143. DOI: 10.1108/14635771011022343.

Aupperle. KE, Dunphy. S, 2003 "Benchmarking financial assessment in the strategy course: a qualitative and quantitative template", Journal of Education for Business, vol. 78, no. 4, pp. 205-212.

Deros. B.M, Tan. J, Ab. Rahman M.N., and N.A.Q.M. Daud, 2011 "An Effective Approach for Benchmarking Implementation", American J. of Engineering and Applied Sciences 4 (2): 288-293, 2011. ISSN 1941-7020, Science Publications

Ertek. G, Ulus. F, Sevinç. M, Şen, 2013 "A new framework for industrial benchmarking”, https:/www.researchgate.net/publication/283525643.

Gustafson. J, 2004 “Purpose-Based Benchmarks", The International Journal of High Performance Computing Applications, Research Article https://doi.org/10.1177/1094342004048540.

Kelessidis. V., "BENCHMARKING", 2000, Report produced for the EC funded project, INNOREGIO: dissemination of innovation management and knowledge techniques, https://www.researchgate.net/.../innoregio_benchmarking-en.pdf

Khurrum S. Bhutta and Faizul Huq, 1999 "Benchmarking: An International Journal", Vol. 6 No. 3, University Press, 1463-5771.

Michael J Col, 2011 "Benchmarking: Contemporary Modalities and Applications", Evaluation Journal of Australasia, Vol. 11, No. 2.

Razmi, J., M. Zairi and Y.F. Jarrar, 2000. "The application of graphical techniques in evaluating benchmarking partners benchmarking”. Int. J., 7: 304-314. DOI: $10.1108 / 14635770010378936$.

Shen, X.X., K.C., Tan and M. Xie, 2000. "Benchmarking in QFD for quality improvement benchmarking”, Int. J., 7: 282-291. DOI: 10.1108/14635770010378918.

Watson, G.H. and C.F. DeYong, 2010. “Design for six sigma”. Caveat emptor. Int. J. Lean Six Sigma, 1: 66-84. DOI: 10.1108/20401461011033176. 